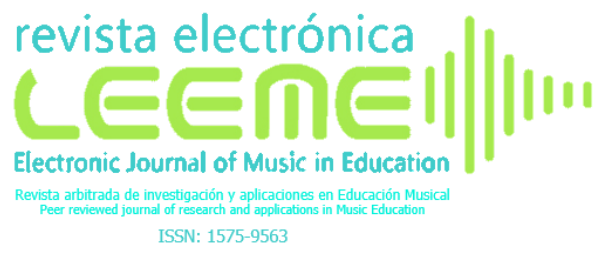

\title{
Características organizativas y didácticas del concierto didáctico. Un estudio de caso sobre la coordinación entre profesorado de Música de Educación Primaria y Secundaria en la ciudad de Manises
}

Organizational and methodological characteristics of the didactic concert. A case study on the coordination between primary and secondary education music teachers in the city of Manises

\author{
María Capel Martín \\ macapelma@gmail.com \\ Máster de Investigación en Didácticas Específicas (Educación Musical) \\ Universidad de Valencia \\ Remigi Morant Navasquillo \\ remigi.morant@uv.es \\ Departamento de Didáctica de la Expresión Musical, Plástica y Corporal \\ Universidad de Valencia
} ORCID: http://orcid.org/0000-0001-5810-2219

doi: 10.7203/LEEME.44.15868

Recibido: 29-09-2019 Aceptado: 25-11-2019. Remigi Morant. Departamento de Didáctica de la Expresión Musical, Plástica y Corporal. Facultad de Magisterio. Universidad de Valencia. Avda. Tarongers, 4. 46022-Valencia. España.

\section{Resumen}

Este estudio de caso plantea, como objetivo principal de investigación, determinar si un concierto didáctico facilita la coordinación entre el profesorado de música de Primaria y Secundaria en la ciudad de Manises. El marco teórico aborda las investigaciones sobre colaboración y coordinación docente, los conciertos didácticos y la transición entre ambas etapas educativas. Un cuestionario, un grupo de discusión y la memoria del grupo de trabajo han revelado las percepciones de los informantes sobre el tema de estudio desde un enfoque cualitativo; y el programa ATLAS.Ti ha facilitado el análisis de datos proporcionando mapas de categorías emergentes. Como conclusión, este proyecto basado en una praxis musical activa, significativa y compartida genera estrategias de aprendizaje colaborativo capaces de mejorar la práctica docente. Aunque hemos detectado dificultades logísticas y administrativas, el grupo de profesores se mantiene cohesionado por afinidades profesionales, por el compromiso por la educación y por la satisfacción de conectar emocionalmente con el alumnado a través de la música.

Palabras clave: Coordinación docente, colaboración docente, concierto didáctico, transición de Primaria a Secundaria, buenas prácticas educativas.

\begin{abstract}
The main goal of this study is to determine which characteristics of didactic concerts, an extracurricular activity at Primary and High Schools in Manises (Spain), facilitate the coordination between Primary and Secondary staffs of the educational centers under consideration. Such coordination endeavors a smooth transition of the students from Primary to Secondary educational stages. The theoretical framework follows the lines of collaboration and teaching coordination, didactic concerts and the transition between the two educational stages. A questionnaire, a focus group, and a memorandum of the aforementioned staff have been used to reveal the perceptions of the informants from a qualitative approach. ATLAS.Ti software allows for a determination of maps of emergent categories. In conclusion, this project based on an active, meaningful, and shared musical praxis generates strategies of collaborative learning that make it possible to improve teaching practice. Although there are logistical and administrative difficulties, the group of teachers remains cohesive as a result of professional affinities, commitment to education, and the satisfaction of connecting emotionally with students by means of the music.
\end{abstract}

Key words: Teacher coordination, teaching collaboration, didactic concert, transition from primary to secondary, good educational practices, useful educational practice. 


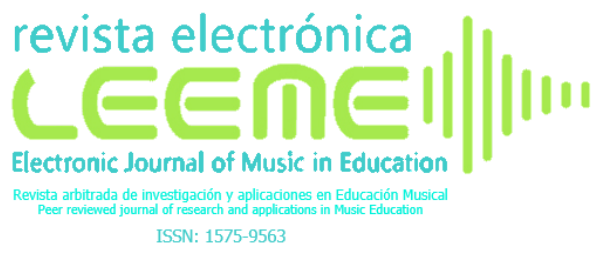

\section{Introducción}

La presente investigación versa sobre un caso concreto de coordinación entre profesorado de música de Educación Primaria y Educación Secundaria de la localidad valenciana de Manises (España). En el curso escolar 2004/2005, profesores y profesoras de música pertenecientes a seis colegios públicos de Primaria y al Instituto de Educación Secundaria José Rodrigo Botet de esta ciudad valenciana, crearon un proyecto musical basado en la organización de un concierto didáctico, siendo los protagonistas los estudiantes del tercer ciclo de Primaria, ESO y Bachillerato. Desde aquel momento y hasta ahora este profesorado mantiene esta propuesta y la dinámica de la coordinación necesaria para llevar a cabo el concierto didáctico ( $\mathrm{CD}$, en adelante). A través de la coordinación generada y mediante reuniones distribuidas a lo largo del curso escolar, se establecen las directrices para su elaboración y preparación. Es en este espacio donde el profesorado intercambia, acuerda y decide, entre otros aspectos, los elementos curriculares compartidos necesarios para su realización. El día del $\mathrm{CD}$, el alumnado colabora para lograr un fin común, compartir y disfrutar de la música. El alumnado de Secundaria, interpreta las piezas del programa del concierto y el alumnado de Primaria participa como espectador activo.

Este $\mathrm{CD}$, como recurso pedagógico musical basado en la práctica musical activa, favorece la escucha, el respeto y el sentimiento colectivo de todos los participantes, compartiendo un repertorio musical compuesto por piezas variadas y contrastadas, aportándoles una gran riqueza auditiva y una idea globalizadora de la música, entendiendo que cada pieza es propia de un contexto determinado y fruto de unas características históricas concretas (Rusinek, 2008). Por otro lado, este grupo docente durante un tiempo decidió vincular esta coordinación a la formación del profesorado (CEFIRE de Valencia) constituyendo el grupo de trabajo "Repercussió". Al finalizar cada curso escolar se presentaba la memoria correspondiente al trabajo realizado a lo largo del año. A lo largo de más de una década, este grupo de trabajo ha alcanzado estabilidad y continuidad. Asimismo, el vínculo personal y profesional creado entre el profesorado de música se presenta consolidado, lo que indica su trascendencia.

En definitiva, el estudio se basa en la descripción de un contexto conocido con el fin de presentar un proyecto musical que se considera de buenas prácticas educativas, ya que su singularidad e idiosincrasia residen en la coordinación docente de la Educación Musical entre dos etapas educativas obligatorias, Primaria y Secundaria. Por todo lo mencionado, por la relevancia científica y social de la cultura de la colaboración como reto para una enseñanza de calidad (Armengol, 2001) y por el interés didáctico y el valor formativo del CD en el área de Educación Musical (Hurtado, 2010), se plantea este estudio de investigación cualitativa. 


\subsection{Preguntas y objetivos de investigación}

Para una mejor comprensión del tema de investigación, se pretende su estudio en profundidad desde la perspectiva del profesorado de Música de Primaria y Secundaria de la ciudad de Manises, perteneciente al mencionado grupo de trabajo. Para ello, es primordial conocer las percepciones de dicho profesorado acerca del $\mathrm{CD}$, considerado como recurso pedagógico musical, para saber hasta qué punto ha supuesto una aportación en beneficio de la coordinación docente entre ambas etapas educativas. Por ello, las preguntas de investigación presentadas orientan hacia las respuestas a obtener de la investigación. La pregunta general de investigación planteada es:

- ¿Cuáles son las características organizativas y didácticas del CD de Manises, planteado como un recurso pedagógico musical que favorece la coordinación entre profesorado de música de Primaria y Secundaria?

De ahí que la anterior pregunta general se operativice en las cuatro siguientes:

- Implementar este CD, ¿facilita la transición de Primaria a Secundaria?

- Implementar este CD, ¿ayuda a garantizar la continuidad curricular?

- Implementar este CD, ¿contribuye desde la formación continua a la mejora profesional?

- ¿Cuáles son las particularidades de este grupo de docentes de música?

Por otra parte, la intención principal del estudio se expresa mediante el siguiente objetivo general de investigación:

- Determinar los factores del CD de Manises que facilitan la coordinación entre profesorado de música de Primaria y Secundaria.

Asimismo, se plantean cuatro de índole más específica:

- Identificar los beneficios e inconvenientes de este CD en la transición entre Primaria y Secundaria.

- Describir los procedimientos necesarios que aseguren la continuidad curricular.

- Identificar las aportaciones que genera la formación continua en el grupo de trabajo de docentes de música.

- Determinar las características de este grupo de docentes de música.

@MARIA CAPEL MARTIN Y REMIGI MORANT NAVASQUILLO. THE CONTENT OF THIS ARTICLE IS THE SOLE RESPONSIBILITY OF THE AUTHORS. THE REVISTA ELECTRÓNICA DE LEEME AND UNIVERSITAT DE VALĖNCIA ARE NOT LIABLE FOR ANY LEGAL ACTIONS THAT MAY ARISE INVOLVING THE ARTICLE'S CONTENT. REVISTA ELECTRÓNICA DE LEEME -LISTA ELECTRÓNICA EUROPEA DE MÚSICA EN LA EDUCACIÓN-HTTP://OJS.UV.ES/INDEX/PHP/LEEME/INDEX. ISSN: 1575-9563. EDITORES: UNIVERSIDAD DE VALENCIA Y JESÚS TEJADA GIMÉNEZ. VISIBILIDAD DE ESTA REVISTA: SCOPUS, EMERGING SOURCES CITATION INDEX (CLARIVATE), EBSCO, CINDOC (CSIC), CITEFACTOR, COPAC, DIALNET, DICE (CSIC), ULRICHS, ESTA REVISTA ESTÁ PUBLICADA CON EL APOYO INSTITUCIONAL DE REDIRIS-CONSEJO SUPERIOR DE INVESTIGACIONES CIENTIFICAS Y ES DE ACCESO LIBRE. CREATIVE COMMONS LICENSE $4.0 \mathrm{BY}$ 


\section{Marco Teórico}

El marco teórico de este estudio se ha construido siguiendo las líneas de investigación de la colaboración y coordinación docente, los conciertos didácticos y de forma tangencial la transición educativa entre Primaria y Secundaria.

La falta de coordinación entre el profesorado de Primaria y Secundaria es un problema frecuente, a pesar de que las etapas de Primaria y Secundaria forman parte de la educación básica obligatoria y la Administración Educativa regula su transición (Pedrera, 2010). Sin embargo, Krichesky y Murillo (2011) analizan la importancia de los intercambios docentes como eje del desarrollo profesional, proponiendo el establecimiento de comunidades profesionales de aprendizaje, con el propósito de mejorar el aprendizaje de los estudiantes. Asimismo, Cañizares, Pomares y González (2015) consideran que es necesaria una coordinación participativa del profesorado a la hora de plantear propuestas pedagógicas para crear proyectos educativos, en los que su desarrollo esté basado tanto en criterios unificadores de la práctica docente, como en modelos que integren los distintos elementos del currículum.

A continuación, se citan varios autores que muestran sus consideraciones con respecto a la coordinación del profesorado de música. Hurtado (2014) afirma que a través de los seminarios o grupos de trabajo se promueve la organización de encuentros musicales, en la modalidad de conciertos didácticos, donde el alumnado puede adoptar el papel de intérprete o participar como público. Morant (2014), analiza la necesidad de coordinación entre Escuelas de Música, Conservatorios, Educación Primaria, Educación Secundaria, Bachillerato y Universidad como una oportunidad de mejora educativa. Este autor considera que la creación de planes de colaboración entre centros educativos y, por ende, con todas las personas implicadas en el proceso educativo, posibilita la innovación, el intercambio de experiencias, la elaboración de materiales y recursos, y la reflexión sobre la praxis docente. Ros (2015) considera que la colaboración entre docentes de música y la formación continua son factores que mejoran su profesión, concretamente en lo que se refiere a la satisfacción personal y a la emancipación profesional, eliminando por tanto el aislamiento de su propio centro. Este autor propone la investigación-acción colaborativa como una herramienta capaz de desarrollar la profesionalización docente musical, basada en el establecimiento de innovaciones metodológicas, en el consenso cooperativo y el análisis reflexivo del profesorado. Por otro lado, Carrillo y Villar (2014) establecen una relación entre la participación en actividades de formación permanente y el perfil profesional del profesorado de música, definiéndola como un estímulo en el desarrollo profesional del docente de música y como una fuente de motivación profesional que repercute en la mejora de habilidades y capacidades personales.

En consecuencia, estas competencias profesionales deseables en el profesorado de música de Educación Primaria y Secundaria, estudiadas por los autores anteriores, podrían 
ayudar en el proceso de transición si los equipos docentes optimizaran los niveles curriculares, organizativos y participativos del alumnado (Martínez y Pinya, 2015). Fidalgo y Barrera (2014) opinan que una de las claves para garantizar un óptimo proceso de transición de Primaria a Secundaria es fomentar más, si cabe, la comunicación entre el profesorado de los colegios y los institutos, estableciendo relaciones de coordinación y colaboración docente basadas en proyectos interinstitucionales que ayuden a regular y mejorar las consecuencias que este paso educativo ejerce sobre los estudiantes. Fabuel (2015), considerando la transición como una oportunidad de desarrollo de las capacidades del discente, plantea mediante mecanismos sinérgicos la búsqueda de elementos comunes en la realización de proyectos educativos. González y González (2015) también consideran que la cooperación y la coordinación entre docentes e instituciones son necesarias en la escuela del siglo XXI. Además, añaden que estas relaciones de coordinación potencian el proceso de transición e impulsan la continuidad curricular.

Antes de finalizar este apartado, se mencionan varios autores que presentan, en sus estudios recientes, las características, particularidades y significaciones que determinan el CD. Sánchez (2016) define el CD como una actividad con intención pedagógica que tiene como eje central la música en vivo. Marín y Domínguez (2015) lo consideran un recurso pedagógico musical diseñado con unos criterios de programación específicos. Ros (2015) señala que el concierto es una herramienta metodológica capaz de fomentar la motivación y de estimular la creatividad, el trabajo cooperativo y la vivencia plena del hecho musical entre los estudiantes de $5^{\mathrm{o}}$ y $6^{\mathrm{o}}$ de Primaria. Castaño (2012) lo determina como práctica musical y motor motivador del proceso educativo, es decir, como "el modo de lograr que los alumnos experimenten formas de hacer y pensar musicales en el marco de un proyecto común, lo que constituye un valioso punto de partida para hacer nacer en ellos actitudes y valores perdurables" (p.46).

\section{Método}

La presente investigación es un estudio de caso de tipo intrínseco según la clasificación de Stake (1998), en el cual el interés de la investigación versa sobre la comprensión y la interpretación de este caso particular de coordinación del grupo de profesorado de Música de Primaria y Secundaria de Manises, en relación a la realización y organización del CD. En esta investigación cualitativa, a través de un diseño emergente, se realiza una descripción de lo que ocurre para conocer la singularidad del caso en sí mismo, buscando su interés y su contexto particular a partir de las expresiones y actividades de las personas participantes, sin pretender producir teoría, ni generalización o representar a otros casos.

Para conseguir un entendimiento óptimo del caso, se ha abordado a los informantes pertenecientes al grupo de trabajo docente, los cuales han mostrado una gran predisposición a participar y a colaborar con su opinión sobre los temas propuestos. Se ha obtenido la 
información de participantes a través de tres instrumentos de recogida de datos cualitativos: un cuestionario de preguntas abiertas que ha sido validado con anterioridad, un grupo de discusión y la memoria de trabajo del grupo de docentes de música.

Para la elaboración del cuestionario se ha diseñado un formato que presenta una introducción, un bloque inicial y un cuerpo principal. En la introducción, se han incluido las instrucciones para cumplimentarlo, así como la fecha límite para mandarlo. En el bloque inicial, se ha recogido la información identificativa de los docentes a los que va dirigida mediante siete cuestiones de índole personal y profesional, aunque anónimo, con el objeto de obtener datos concretos del sujeto necesarios para la buena interpretación de sus respuestas. En el cuerpo principal se han tenido en cuenta los cuatro objetivos específicos de la investigación que han ayudado a formular la batería de nueve preguntas de carácter abierto con la intención de recibir de los y las informantes respuestas amplias y libres para así conocer sus circunstancias, sus ideas generales, sus valoraciones y opiniones. Las preguntas han sido distribuidas en tres bloques temáticos dotando al cuestionario de coherencia interna.

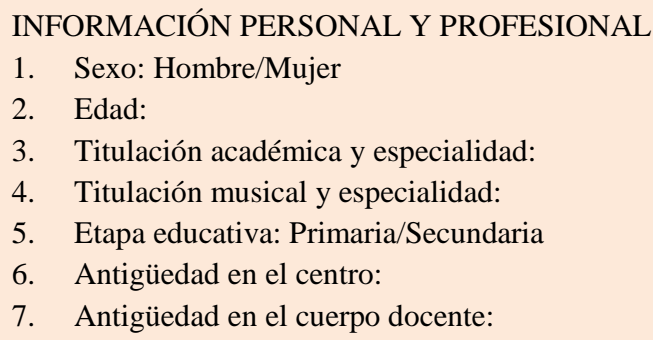

\section{SOBRE LA COORDINACIÓN ENTRE PRIMARIA Y SECUNDARIA}

1. ¿De qué manera crees que el recurso pedagógico del concierto didáctico podría favorecer la coordinación entre el profesorado de música de Primaria y Secundaria?

2. ¿Cuáles crees que son las debilidades y fortalezas de la coordinación entre el profesorado de música de Primaria y Secundaria?

3. ¿Cómo valorarías tu participación en el grupo de trabajo de profesorado de música de Primaria y Secundaria en Manises?

4. ¿La participación en el grupo de trabajo de profesorado de música de Primaria y Secundaria en Manises, crees que tiene algún efecto sobre tu práctica docente?

\section{SOBRE LA TRANSICIÓN DE PRIMARIA A SECUNDARIA}

5. ¿Cuáles crees que podrían ser los beneficios o inconvenientes de implementar el recurso pedagógico del concierto didáctico en la transición de Primaria a Secundaria? 


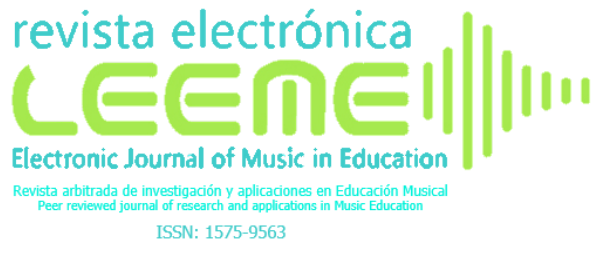

6. Pensando en el recurso pedagógico del concierto didáctico, ¿cómo crees que los objetivos planteados, como elemento curricular compartido, podrían favorecer la continuidad curricular de Primaria a Secundaria?

7. Pensando en el recurso pedagógico del concierto didáctico, ¿cómo crees que los contenidos trabajados, como elemento curricular compartido, podrían garantizar la continuidad curricular de Primaria a Secundaria?

8. Pensando en el recurso pedagógico del concierto didáctico, ¿cómo crees que la metodología aplicada, como elemento curricular compartido, podría fomentar la continuidad curricular de Primaria a Secundaria?

9. Pensando en el recurso pedagógico del concierto didáctico, ¿cómo crees que la evaluación utilizada, como elemento curricular compartido, podría impulsar la continuidad curricular de Primaria a Secundaria?

Figura 1. Apartados del cuestionario empleado

Para la elaboración del grupo de discusión, se consideraron a priori tres temas generales con la idea de que pudieran surgir otros subtemas, según fuera desarrollándose la conversación: el concierto didáctico y la coordinación docente, el concierto didáctico y la transición de Primaria a Secundaria, el concierto didáctico y la continuidad curricular.

El análisis de la memoria del grupo de trabajo se llevó a cabo a partir de un documento reelaborado a partir de la primera memoria realizada en el curso escolar 2004-2005.

Para la validación del cuestionario, se ha utilizado un sistema de validación mixto que ha combinado juicio de expertos y juicio por parte de sujetos. En la primera fase se ha diseñado un cuestionario provisional que ha sido presentado al profesorado del Departamento de Didáctica de la Expresión Musical, Plástica y Corporal de la Facultad de Magisterio de Valencia, realizando una primera validación de contenidos. En el proceso de revisión especializada del cuestionario, propusieron la eliminación de algunas preguntas y sugirieron algunos cambios en la enunciación de otras (fundamentalmente por problemas de sesgo), así como la distribución de las preguntas en tres campos temáticos diferenciados. Estos motivos condujeron a realizar las modificaciones oportunas. En la segunda fase, se incorporaron al juicio 4 maestros/as de características semejantes a los encuestados, pero que ejercen su docencia en poblaciones distintas a Manises. A estos docentes, se les solicitó que realizaran los aportes que consideraran oportunos para la mejora del cuestionario, y que emitieran un juicio crítico sobre la claridad y la precisión de las nueve preguntas. En la tercera fase, se elaboró definitivamente el cuestionario, en el que se realizaron los últimos ajustes oportunos, basados en la ordenación y clasificación de las nueve preguntas definitivas en tres bloques temáticos. Concluido el proceso de validación por parte del juicio de expertos, se confeccionó el cuestionario definitivo.

La validación del grupo de discusión se realizó de manera semejante, aprobando el grupo de expertos los tres bloques temáticos apuntados.

El cuestionario lo han cumplimentado un total de 7 profesores ( 5 docentes de música de Primaria y 2 docentes de música de Secundaria), ubicados todos en Manises (Valencia). En 


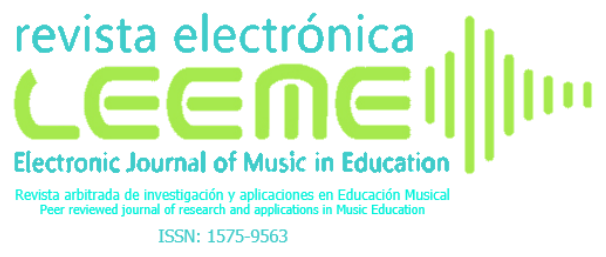

cuanto al grupo de discusión, ha estado conformado por cuatro profesores: 2 de música de Primaria y 2 de música de Secundaria.

Posteriormente, se ha realizado un análisis inductivo de todos los datos generados en el que las categorías emergentes han aflorado por reiteración de códigos, con el fin de describir este caso concreto. Según Hernández-Sampieri et al. (2014), se ha realizado una codificación abierta o de primer nivel, consistente en identificar y relacionar las unidades de análisis codificándolas en categorías emergentes. Es decir, en la codificación cualitativa se han relacionado y comparado las unidades de análisis provenientes de los datos de las tres fuentes mencionadas anteriormente. Este procedimiento ha permitido asignar unos códigos, los cuales nos han permitido obtener una serie de categorías emergentes por medio de procesos de refinado de los mismos. A continuación, en la codificación axial de las categorías o segundo nivel se han comparado, se han descrito y se han interpretado todas las categorías emergentes entre sí para agruparlas por familias de hipercategorías. De este modo, se ha logrado la identificación de las categorías emergentes y sus interconexiones y con ello la creación de cinco hipercategorías. Para el proceso inductivo de análisis de contenido semántico descrito anteriormente se ha utilizado el programa informático Atlas.Ti, herramienta que ha facilitado llegar a cinco mapas de categorías. Las cinco hipercategorías creadas se corresponden con las preguntas de la investigación y se han denominado de la siguiente manera: "Concierto didáctico-coordinación", "Transición", "Continuidad curricular", "Formación continua" y "Perfil del grupo docente". Finalmente, se ha generado el desarrollo de un análisis global que ha permitido la explicación y la descripción de cada uno de los cinco mapas de categorías emergentes con sus correspondientes hipercategorías.

\subsection{Análisis de los resultados}

En este apartado, se presentan los resultados obtenidos, tomando como base el análisis global de los datos extraídos de los tres instrumentos utilizados en el estudio. A cada instrumento se le ha asignado un código: el cuestionario se corresponde con el código P1, el grupo de discusión con el código P2 y la memoria del grupo de trabajo con el código P3. La finalidad de utilizar dichos códigos es mostrar, de una forma clara, la relación entre la categoría emergente y el instrumento de donde emerge. Seguidamente, se expone una síntesis integradora de cada uno de los cinco mapas de categorías emergentes con su hipercategoría correspondiente: 


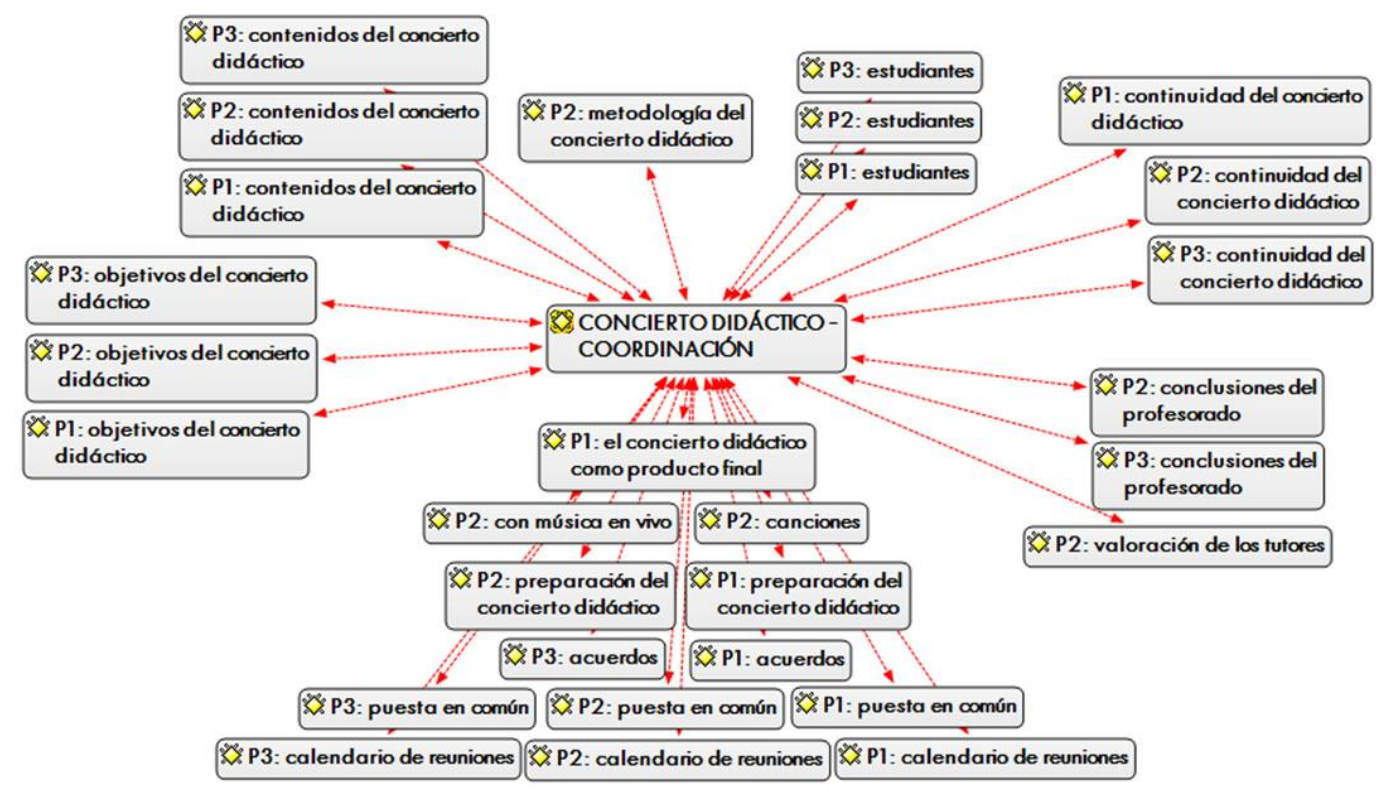

Figura 2. Mapa de la hipercategoría "Concierto Didáctico-Coordinación"

En el mapa de la Figura 2, se presentan las categorías emergentes que muestran los factores que favorecen la coordinación y, por ende, la creación del grupo de trabajo de docentes de música de Primaria y Secundaria. El CD de Manises, se define como el producto final compartido tras un proceso de enseñanza-aprendizaje previo en el aula. Es una experiencia vivida en la que el alumnado de Primaria y Secundaria interpretan conjuntamente un repertorio de canciones, haciendo música en vivo. Para organizar este CD se genera la necesidad de una coordinación docente. El desarrollo de las dinámicas dialógicas, promueve que las reuniones se contemplen como un espacio abierto y flexible, en las que se da cabida a todas las ideas y propuestas que van surgiendo. Por último, la adopción de acuerdos por parte de todos los miembros del grupo en favor del logro de un objetivo común (implementar esta actividad musical), facilita la preparación del CD y, por tanto, la coordinación entre docentes.

Asimismo, el CD presenta otros factores que fomentan la coordinación. Por un lado, la formulación de los objetivos que determinan la acción docente y discente. Por otro, el establecimiento de los contenidos a trabajar en cada una de las canciones. También, la búsqueda de la metodología de trabajo docente a aplicar, en este caso, basada en la participación activa de la música, y, por último, la evaluación utilizada tanto en el proceso de enseñanza aprendizaje, como al finalizar el proyecto.

Otro factor a considerar es el estudiante, tanto de Primaria como de Secundaria. Para llevar a cabo el CD, cada grupo de estudiantes tiene roles diferenciados y el grupo de profesores determina sus funciones. El alumnado de Educación Primaria es un público activo, participa en 
algunas canciones realizando las cuatro acciones básicas musicales (cantar, bailar, escuchar y tocar instrumentos de percusión o corporales), y los de Secundaria forman el equipo de percusión e interpretan todo el repertorio. Por lo que respecta a las características comunes, se destaca que todos comparten y sienten la vivencia de la música.

El siguiente factor es el que alude a la continuidad del CD. Dicha continuidad es debida a las circunstancias laborales del profesorado de Primaria y Secundaria, ya que se trata de profesorado definitivo en cada centro educativo, lo que favorece la participación continua y dilatada en el tiempo en el grupo de coordinación.

El último factor hace referencia a las conclusiones y valoraciones del profesorado de música y de los tutores. La organización del CD genera ilusión en el grupo de trabajo. Igualmente, la realización del CD refuerza la importancia y el valor de la música, en este caso, como eje vertebrador de experiencias musicales. Por último, el CD impulsa la importancia del alumno en el proceso educativo, siendo éste el protagonista absoluto. En cuanto a la opinión del profesorado tutor, valora de manera positiva esta actividad, mostrando la importancia de desarrollar este trabajo dentro de la dinámica del centro en beneficio del educando, de modo que alientan el trabajo del grupo de coordinación, animándolos a que continúen trabajando, planteándose nuevos retos y conciertos.

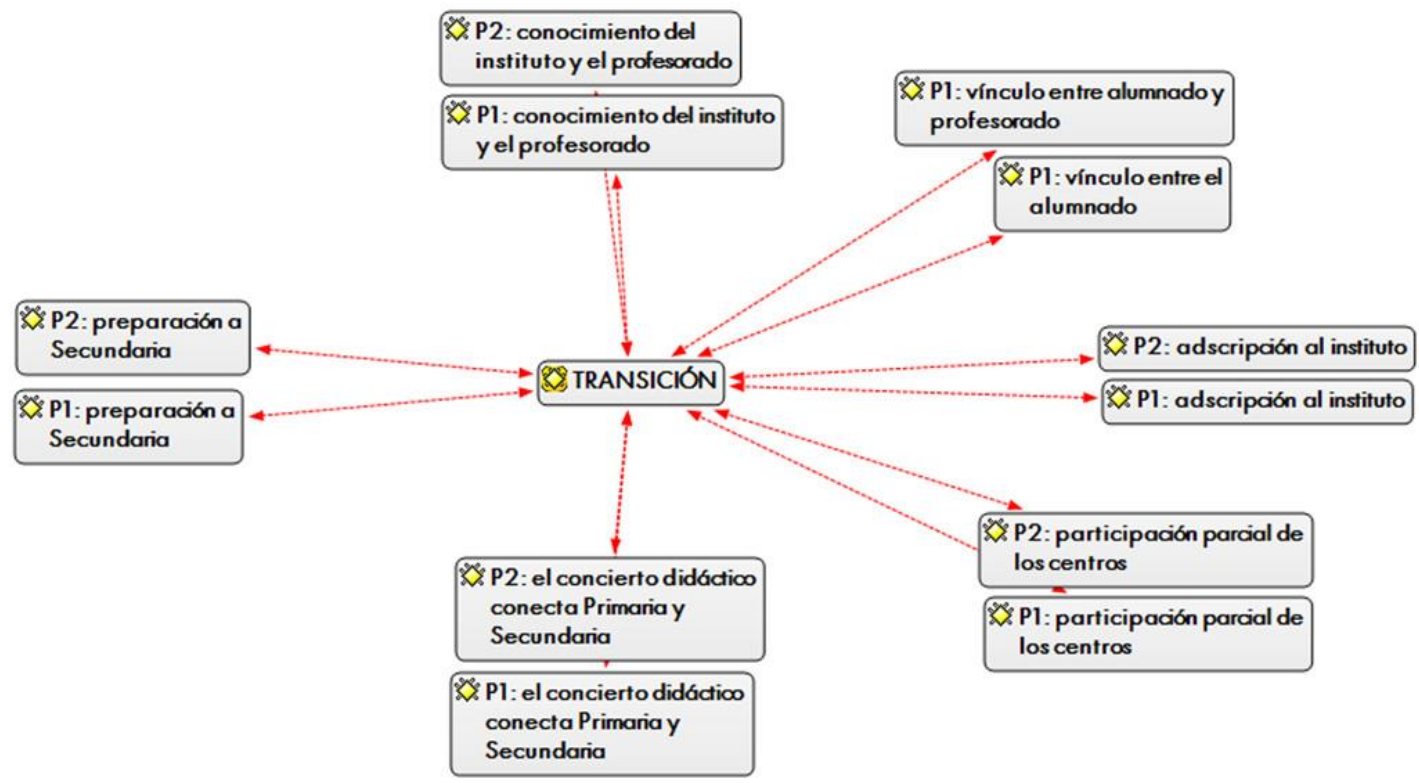

Figura 3. Mapa de la hipercategoría "Transición" 


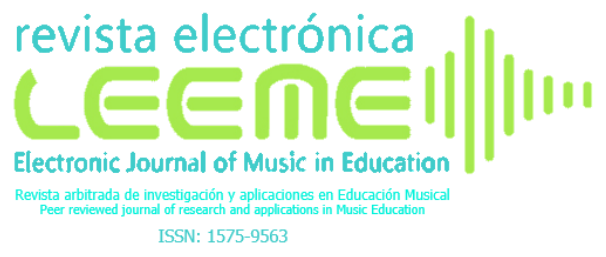

En el mapa de la Figura 3, se presentan las categorías emergentes que señalan los beneficios y las debilidades del CD en el paso de Primaria a Secundaria. El CD conecta Primaria y Secundaria, tanto por el alumnado participante como por el profesorado que lo organiza. Es una preparación para Secundaria, ya que los estudiantes de Primaria tienen la oportunidad de conocer de antemano las instalaciones y el profesorado, lo que van a estudiar y cómo lo van a estudiar el curso siguiente en el instituto, es decir, los elementos curriculares, la metodología aplicada, la manera de evaluar, y los materiales utilizados.

El CD genera vínculos entre el alumnado y el profesorado y entre el propio alumnado. Gracias a la música, se desarrolla, por un lado, un nexo de confianza y de conocimiento mutuo entre el alumnado y el profesorado de ambas etapas educativas. Y, por otro, se genera una conexión entre el alumnado de Primaria y Secundaria en el logro de un fin común, es decir, se desarrolla una actitud de colaboración en el momento de hacer y disfrutar de la música, compartiendo.

El CD, en líneas generales, facilita la transición musical a Secundaria. Sin embargo, hay que puntualizar que los beneficios de la transición son totales para el alumnado adscrito a este instituto y para los no adscritos, pero que desean matricularse en él.

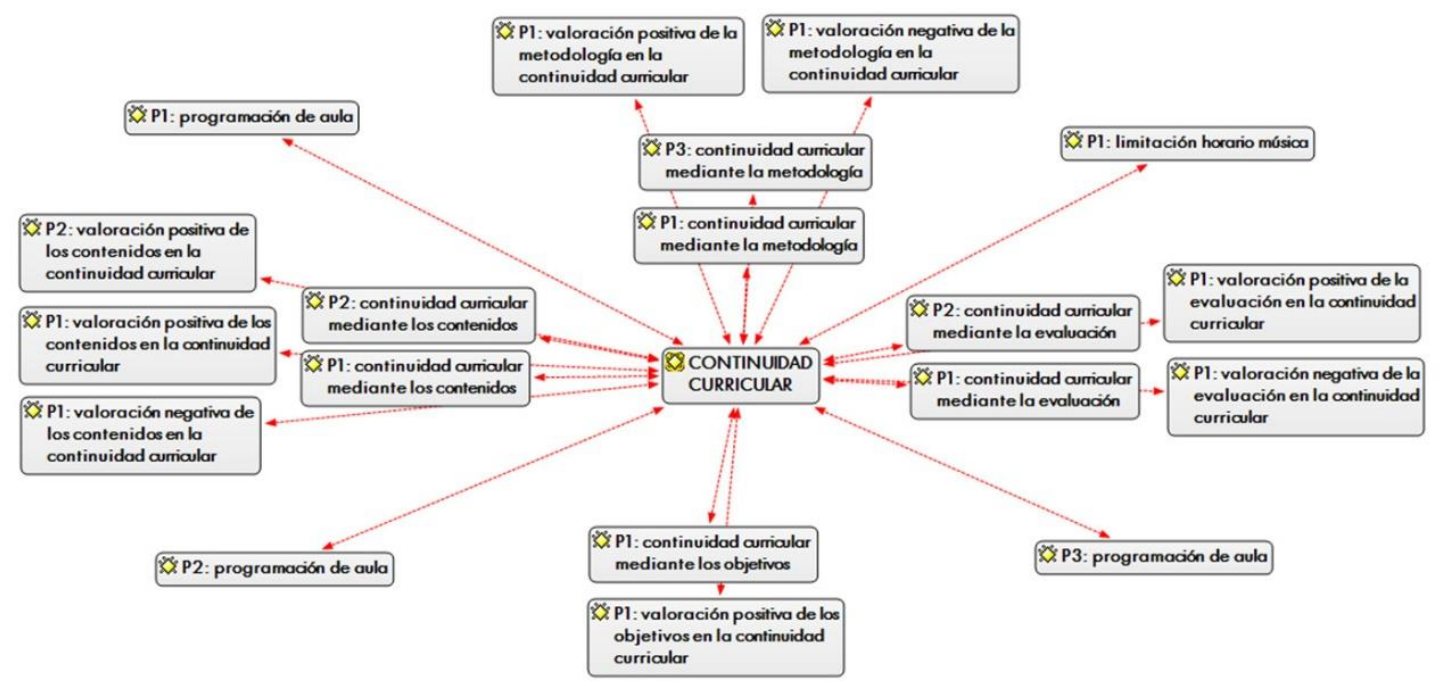

Figura 4. Mapa de la hipercategoría "Continuidad Curricular"

En el mapa de la Figura 4, se presentan las categorías emergentes sobre los elementos curriculares compartidos y su continuidad curricular. El trabajo de coordinación de este grupo docente se desarrolla teniendo en cuenta varios aspectos: el análisis de los bloques de contenido y su adaptación a los contenidos del concierto, la adecuación de la metodología a las exigencias 
del concierto, y la evaluación procedimental individual y grupal de clase y la del día del concierto. Por tanto, la elaboración del CD garantiza la continuidad curricular, ya que los elementos curriculares son compartidos y comunes entre Primaria y Secundaria.

Sin embargo, se presentan valoraciones que explican que el CD se podría plantear como un proyecto más amplio en el que los objetivos, contenidos, metodología y evaluación debieran formularse de una forma global y coordinada. Por otro lado, las valoraciones indican que la programación de aula de cada profesor ha de contemplar el proyecto del $\mathrm{CD}$, de manera que los elementos curriculares vigentes estén vinculados con las actividades propuestas para implementar el repertorio del concierto.

La última valoración trata el tema del insuficiente número de horas de clase de música en $6^{\circ}$ de Primaria, que implica una limitación en la calidad del trabajo del profesorado de Primaria con sus estudiantes.

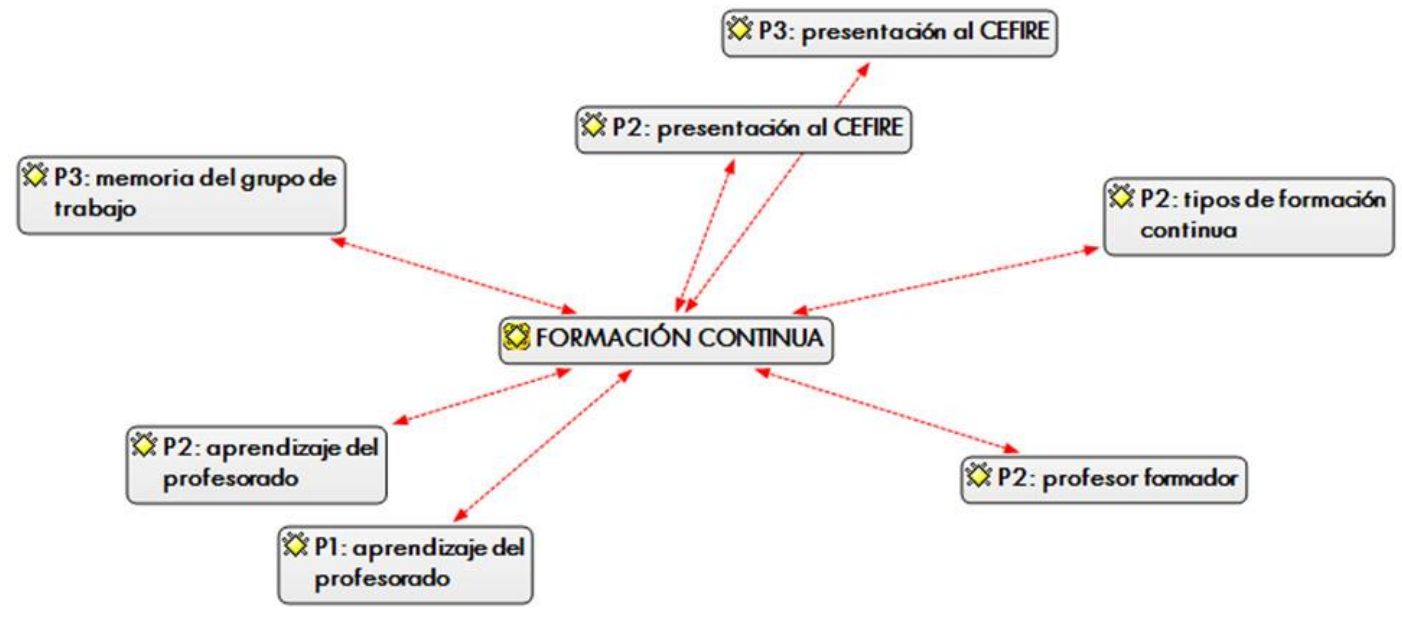

Figura 5. Mapa de la hipercategoría "Formación Continua"

El mapa de la Figura 5 presenta las categorías emergentes que indican los efectos que genera la formación continua en el grupo de trabajo y las aportaciones que comporta. La formación continua se produce en el grupo docente a través de la elaboración y la presentación al CEFIRE de Valencia de la memoria del grupo de trabajo realizado. El profesorado de este grupo de trabajo que prepara y organiza el CD está inmerso en un proceso de enseñanzaaprendizaje basado en la interacción, el feed-back y la reflexión entre los miembros del equipo. Es decir, a través de la realización del proyecto del $\mathrm{CD}$ este profesorado participa y recibe una

@MARIA CAPEL MARTIN Y REMIGI MORANT NAVASQUILLO. THE CONTENT OF THIS ARTICLE IS THE SOLE RESPONSIBILITY OF THE AUTHORS. THE REVISTA ELECTRÓNICA DE LEEME AND UNIVERSITAT DE VALĖNCIA ARE NOT LIABLE FOR ANY LEGAL ACTIONS THAT MAY ARISE INVOLVING THE ARTICLE'S CONTENT. REVISTA ELECTRÓNICA DE LEEME -LISTA ELECTRÓNICA EUROPEA DE MÚSICA EN LA EDUCACIÓN-HTTP://OJS.UV.ES/INDEX/PHP/LEEME/INDEX. ISSN: 1575-9563. EDITORES: UNIVERSIDAD DE VALENCIA Y JESÚS TEJADA GIMÉNEZ. VISIBILIDAD DE ESTA REVISTA: SCOPUS, EMERGING SOURCES CITATION INDEX (CLARIVATE), EBSCO, CINDOC (CSIC), CITEFACTOR, COPAC, DIALNET, DICE (CSIC), DOAJ, E-REVISTAS (CSIC), EBSCO PREMIER, ERIH+, GALE CENGAGE LEARNING, IN-RECS, IRESIE, LATINDEX, MIAR, OCLC WORLDCAT, RESH, REDIB, RILM CORE JOURNALS, SUDTI,
ULRICHS, ESTA REVISTA ESTA PUBLICADA CON EL APOYO INSTITUCIONAL DE REDIRISCONSEJO SUPERIOR DE INVESTIGACIONES CIENTIFICAS Y ES DE ACCESO LIBRE. CREATIVE COMMONS LICENSE 4.0 BY 
formación continua. En este espacio de enriquecimiento profesional, el profesorado se beneficia de dos tipos de formación: la grupal, en la que aprende dentro de un grupo de trabajo, y la individual como docente de su aula, ya que aplica lo aprendido en el grupo mejorando su práctica docente.

Por último, aparece dentro del grupo de trabajo un docente formador, que ha impartido cursos de formación a profesores para enseñar y explicar la metodología y otros aspectos en la realización de propuestas didácticas basadas en la instrumentación.

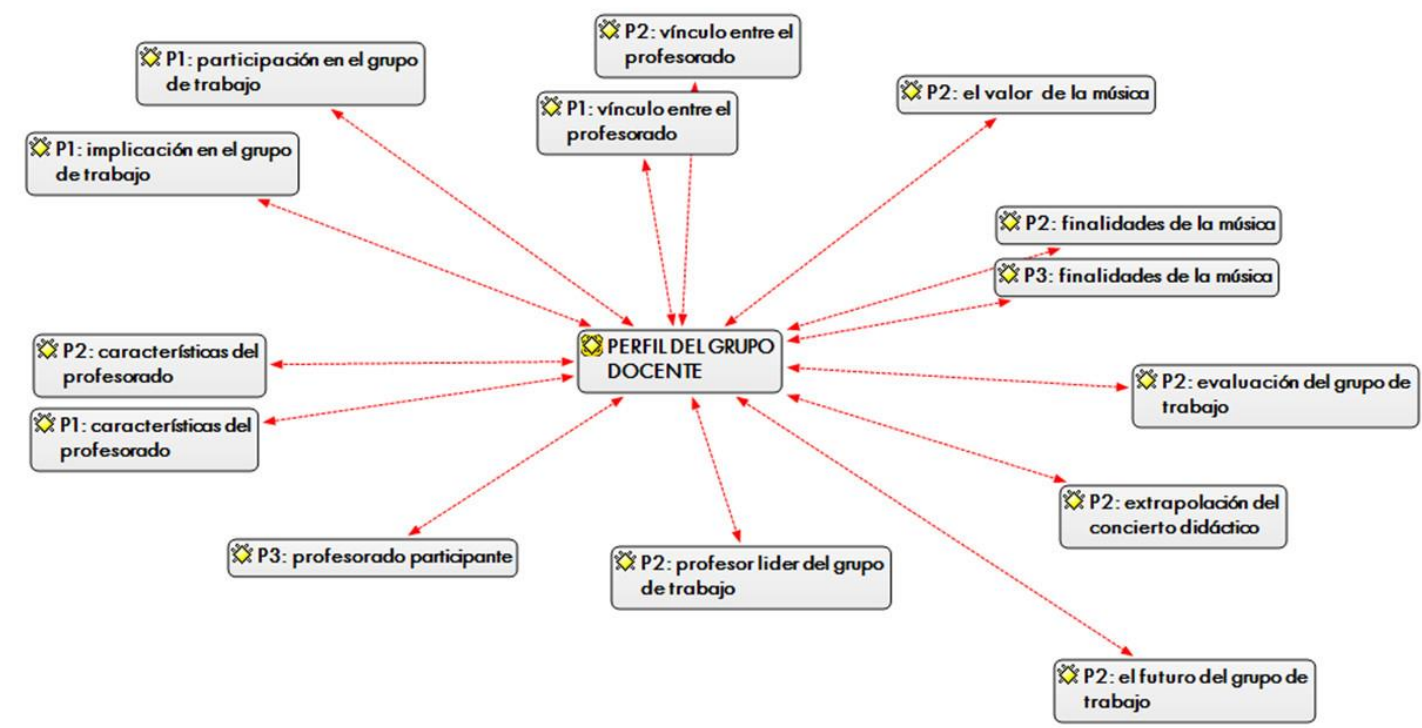

Figura 6. Mapa de la hipercategoría "Perfil del Grupo Docente"

El mapa de la Fig. 6 presenta las categorías emergentes enumerando las principales características que definen el grupo docente. El profesorado participante en este grupo de trabajo imparte clases de música y pertenece a Primaria y Secundaria. Es un grupo compuesto por hombres y mujeres con una dilatada trayectoria profesional y experiencia docente, con antigüedad tanto en el cuerpo docente como en el centro educativo, lo que proporciona estabilidad y continuidad al grupo de trabajo.

En este grupo docente, destaca el profesorado líder del grupo de trabajo, ya que fue el que propuso este proyecto al resto de sus compañeros y compañeras de música de la localidad. Este docente inició esta idea movido por la inquietud de conocer al alumnado que iba a recibir de Primaria y por la decisión de eliminar obstáculos y dificultades en la incorporación de este alumnado a Secundaria.

La implicación y la participación en el grupo de trabajo dependen de cada profesor. En general, la valoración es que existe una implicación y participación total, completa y activa, 
mostrando interés y conformidad en los asuntos tratados. El vínculo entre el profesorado es estrecho y va más allá de lo meramente profesional. La continuidad en el tiempo, el compromiso, la responsabilidad y el propio carácter entusiasta, positivo y respetuoso de estas personas hacen que el grupo esté cohesionado en la realización de este proyecto. Es un profesorado comprometido en general con la educación y en particular con la música. Un profesorado que es consciente del valor educativo de la música y que juega un papel activo tanto en la clase con su alumnado como en el grupo de trabajo. La ilusión y su esmerada dedicación en su trabajo le confieren ese grado de responsabilidad que hace que el CD sea un éxito. Es por ello que la importancia que le otorga este grupo docente a la música determina que su trabajo sea, si cabe, más riguroso e innovador.

La evaluación del grupo de trabajo es positiva, basada en la reflexión y considerando que la coordinación es fundamental para llevar a cabo la preparación y realización del CD. En cuanto a la extrapolación del $\mathrm{CD}$, el grupo docente considera que este proyecto se podría desarrollar en otras localidades y por otros docentes de música. Los profesores argumentan que la valoración es positiva y además existe el apoyo de la administración educativa.

\section{Discusión y conclusiones}

En este apartado, se establece un debate teórico que relaciona los resultados obtenidos en este estudio con los resultados de otros trabajos empíricos abordados en la revisión de la literatura relacionada con el objeto de investigación. Asimismo, se hace referencia a las limitaciones, abriendo perspectivas a futuras investigaciones que pueden profundizar y dar continuidad al estudio realizado. Exponemos las conclusiones del presente estudio que dan respuesta a los interrogantes iniciales y objetivos de investigación formulados.

En referencia al objetivo de investigación general, referido a los factores del CD que facilitan la coordinación entre profesorado de música de Primaria y Secundaria, son la preparación y la organización del CD los que generan la necesidad de una coordinación práctica y participativa del profesorado. Esta coordinación docente está basada en el planteamiento de una propuesta pedagógica con el objetivo común y compartido de crear un proyecto educativo musical llamado "Concierto Didáctico" (Cañizares, Pomares y González, 2015). Dicho proyecto está contextualizado en el entorno social y cultural de los centros de la localidad (Escudero, 2008) arraigando en el alumnado el disfrute y el valor de la música y la pasión por ella. Este grupo de trabajo docente de coordinación externa posibilita, entre otros aspectos, la reflexión sobre la praxis docente, la cual es indispensable en la continuidad, la innovación y la mejora de este grupo de profesorado de música (Morant, 2014). Al igual que afirma Díaz (2004), esta coordinación se define como una propuesta de acción docente compartida en la que se desarrollan estrategias de colaboración activa en la que están presentes la motivación y la ilusión entre el profesorado. Como considera Hargreaves (1996) cuando habla de la 


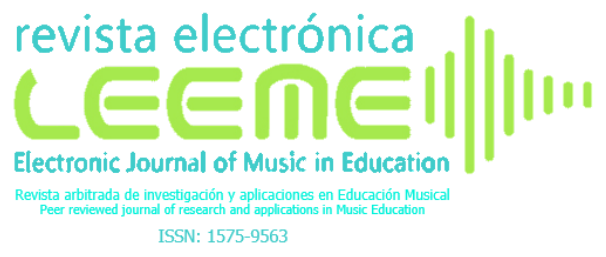

colaboración como estrategia, en este equipo docente se establecen vínculos colegiados y personales positivos, que incitan al compromiso por la educación, al cuestionamiento de su trabajo diario y al desafío por experimentar nuevas formas de hacer música. Asimismo, y tal y como afirman Escudero (2008) y Montero (2011) a la confianza en uno mismo y en los demás. Además de la preparación y la organización del CD, un tercer factor es el propio desarrollo del CD que, como analizan Krichesky y Murillo (2011), produce intercambios docentes basados en el aporte y compartición de ideas, conocimientos y recursos que repercuten positivamente en la práctica docente, generando tanto cambios organizativos y metodológicos, como emocionales. A partir de la puesta en común, el grupo docente adopta acuerdos que implican la formulación de criterios unificadores de su propia práctica y de los elementos del currículum, tal y como apuntan Cañizares, Pomares y González (2015).

Este grupo de trabajo de docentes de música como promotor de la preparación, organización y desarrollo de este CD (Hurtado, 2014) realiza reuniones de coordinación que se convierten en espacios de enriquecimiento personal y profesional (López, 2004). Asimismo, en ellas se establecen relaciones óptimas y fructíferas entre el profesorado (Hurtado, 2004). Igualmente, se promueve la unidad y la cohesión de grupo, la capacidad de aprendizaje y la implicación en el trabajo diario, la ilusión y la idea de una visión futura de la educación (Escudero, 2008). En relación a las reuniones de coordinación y a pesar de lo que afirma Pedrera (2010) sobre la distancia entre los centros educativos y la incompatibilidad horaria lectiva y laboral entre profesorado de Primaria y Secundaria, estos factores no son motivo de descoordinación en este grupo docente. Al contrario, el grupo docente señala que el establecimiento de un calendario de reuniones a principio de curso solventa esta debilidad en la coordinación. Por tanto, el CD de Manises planteado como un recurso pedagógico musical provoca sinergias entre el profesorado (Antúnez, 1999) para colaborar en la preparación, organización y desarrollo de esta actividad, favoreciendo la coordinación docente entre ambas etapas educativas.

En cuanto al primer objetivo específico, y con respecto a los beneficios del CD en la transición de Primaria a Secundaria, el CD se muestra como una preparación para Secundaria, ya que este equipo de trabajo docente adapta y consensua la metodología aplicada con el objeto de aproximarla y relacionarla con las dos etapas y así facilitar el aprendizaje al alumnado (Fidalgo y Barrera, 2014). Otro beneficio del CD es que éste conecta Primaria y Secundaria. A través de la vivencia de esta experiencia musical se generan vínculos entre los propios estudiantes de ambas etapas educativas. Igualmente, se desarrollan vínculos entre los docentes y los discentes de ambas etapas. El profesorado se presenta como nexo de unión entre el alumnado y el centro educativo al que acudirán en Secundaria. Por tanto, las consecuencias son beneficiosas, tanto por la valoración positiva de la asignatura de música por parte del alumnado, como en el deseo de éstos de continuar su educación en el instituto que le ha brindado la oportunidad de vivir esta experiencia musical. 
En relación a la conexión entre etapas y de acuerdo con Fabuel (2015), quien considera la transición como una oportunidad de desarrollo de las capacidades del discente, el grupo de trabajo docente señala la necesidad de conocer y analizar qué y cómo se trabaja en Primaria con el objeto de conectar con el alumnado y así conocer sus intereses y dudas como herramientas clave para el éxito escolar. Al igual que González y González (2015), el grupo docente también considera las relaciones de coordinación potenciadoras del proceso de transición y de la continuidad curricular, sin embargo, existen inconvenientes que dificultan el logro de una transición suave y fácil. Por un lado, consensuar los objetivos de la transición es complicado por la falta de tiempo y porque no es una cuestión prioritaria en este equipo docente. Por otro lado, la falta de predisposición del resto de centros docentes de la localidad a querer participar en este proyecto educativo. En consecuencia, solo el alumnado de los centros de Primaria adscritos al instituto donde se realiza el CD se benefician del proceso de transición musical en su totalidad. Paradójicamente, existe cierto estudiantado adscrito a su instituto de referencia (el cual no es donde se realiza el CD) que muestra su voluntad de matricularse en este instituto motivado por la relación musical y emocional desarrollada y porque son estudiantes de música. Por tanto, si los equipos docentes de ambas etapas optimizaran los niveles curriculares, organizativos y participativos del alumnado como afirman Martínez y Pinya (2015), el proceso de transición estaría consolidado. La transición como preparación para Secundaria se ve favorecida, por un lado, por la voluntad personal y profesional de desarrollar este proyecto que integra instituciones educativas y especialistas de Música (Hurtado, 2010), y, por otro, por el desarrollo tanto en el aula como el día del CD como producto final de vínculos emocionales entre los docentes y los discentes de ambas etapas educativas (Gimeno, 1996).

En referencia al segundo objetivo específico, con respecto a si el CD ayuda a garantizar la continuidad curricular, el grupo docente parte de dos consideraciones: por un lado, opinan que el CD es una actividad con clara intención pedagógica (Sánchez, 2016) realizada con música en vivo (Neuman, 2004); por otro, apuntan que el concierto es didáctico porque hay un trabajo previo de preparación en las aulas, es decir, de ensayo (Hurtado, 2010; Rusinek, 2002). Así pues, el grupo docente considera que el procedimiento necesario para que se asegure la continuidad curricular es partir de un proyecto globalizador que forme parte a su vez de la programación de aula de cada docente (Hurtado, 2010; Ros, 2015). Igualmente, el grupo docente afirma que el $\mathrm{CD}$ ha de estar diseñado con unos criterios de programación específicos (Marín y Domínguez, 2015). Así pues, la tarea del grupo docente se basa en dotar de unidad y coherencia a todos los elementos curriculares compartidos y comunes en favor del aprendizaje significativo y de calidad del alumnado de Primaria y Secundaria (Rusinek, 2004). Por tanto, esta circunstancia implica, desde el acuerdo y el consenso docente, la formulación de objetivos y contenidos amplios (relacionados con el logro del producto final) y motivadores e interesantes para el alumnado (Hurtado, 2010). Asimismo, conlleva que la metodología a aplicar ha de ser vivencial, práctica (Castaño, 2012) y participativa (Rusinek, 2002) adaptándola al alumnado, fomentando el trabajo autónomo y creativo.

@MARIA CAPEL MARTIN Y REMIGI MORANT NAVASQUILLO. THE CONTENT OF THIS ARTICLE IS THE SOLE RESPONSIBILITY OF THE AUTHORS. THE REVISTA ELECTRÓNICA DE LEEME AND UNIVERSITAT DE VALĖNCIA ARE NOT LIABLE FOR ANY LEGAL ACTIONS THAT MAY ARISE INVOLVING THE ARTICLE'S CONTENT. REVISTA ELECTRÓNICA DE LEEME -LISTA ELECTRÓNICA EUROPEA DE MÚSICA EN LA EDUCACIÓN.HTTP://OJS.UV.ES/INDEX/PHP/LEEME/INDEX. ISSN: 1575-9563. EDITORES: UNIVERSIDAD DE VALENCIA Y JESÚS TEJADA GIMENEZ. VISIBILIDAD DE ESTA REVISTA: SCOPUS, EMERGING SOURCES CITATION INDEX (CLARIVATE), EBSCO, CINDOC (CSIC), CITEFACTOR, COPAC, DIALNET, DICE (CSIC), ULRICHS, ESTA REVISTA ESTÁ PUBLICADA CON EL APOYO INSTITUCIONAL DE REDIRIS-CONSEJO SUPERIOR DE INVESTIGACIONES CIENTIFICAS Y ES DE ACCESO LIBRE. CREATIVE COMMONS LICENSE $4.0 \mathrm{BY}$ 
Este grupo docente considera las estrategias metodológicas que plantea Fabuel (2015) como líneas de coherencia para el éxito escolar, es decir, para que el alumno sea competente y disfrute de la actividad. En relación a la evaluación, el grupo de trabajo docente considera que ésta ha de ser procedimental, fomentando el espíritu crítico del alumno y la autoevaluación, valorando las actividades, tanto individuales como colectivas. Sin embargo, el grupo de trabajo realiza varias apreciaciones que dificultan la continuidad curricular. Por un lado, las diferencias de funcionamiento de cada centro y la diversidad de alumnado; y, por otro, la falta de una mayor conexión metodológica entre las etapas.

Cabe destacar que la gran cantidad de contenidos curriculares a trabajar, unido al hecho del insuficiente número de horas del que dispone el profesorado para impartir la clase de Música en $6^{\circ}$ de Primaria, repercute en el desarrollo óptimo del trabajo cooperativo en el aula y obstaculiza la cohesión grupal en el alumnado (Hurtado, 2010). Y en último lugar, el criterio de calidad y musicalidad del CD difiere entre los miembros del grupo de trabajo, con lo que la experiencia musical puede ser aceptada, entendida y valorada de manera diferente.

En relación al tercer objetivo específico de la investigación, la realización de este CD aporta varias mejoras desde la formación continua por dos motivos fundamentales: la vinculación establecida con el CEFIRE de Valencia durante varios años y el hecho de que el grupo de coordinación se muestra como una oportunidad de formación docente (Escudero, 2008).

Además, pertenecer a este grupo de trabajo genera un estímulo en el desarrollo profesional del docente de música, motivándole profesionalmente y repercutiendo en la mejora de sus habilidades y capacidades personales (Carrillo y Villar, 2014). Asimismo, dado que en el grupo de coordinación el aprendizaje se desarrolla de una manera flexible por la confianza creada, existe un favorecimiento en la mejora profesional de todos los miembros del grupo, lo que genera el desarrollo de prácticas innovadoras y nuevos desafíos (Marcelo, 2001).

En cuanto al cuarto objetivo específico de la investigación, este grupo docente se caracteriza por ser activo, reflexivo, crítico y perfeccionista con su tarea docente como define Hargreaves (1996). Asimismo, este profesorado se caracteriza por su responsabilidad en el trabajo, en la disposición y capacidad de diseñar y desarrollar proyectos de trabajo grupales relacionados con los intereses de los estudiantes, siendo el objetivo común la participación activa en los contextos sociales, escolares y de aula (Reyes, 2005). Además, son docentes comprometidos, implicados y vinculados con la educación en general y con la educación musical en particular, dándole el valor, la importancia y la dignidad que le corresponde a la música (Hurtado, 2010; Pastor, 2004). Igualmente, son conscientes del apoyo y el reconocimiento que los profesores tutores demuestran por la labor cultural realizada en los centros docentes de la localidad (Hurtado, 2010). Por tanto, este grupo docente está caracterizado por una relación de confianza y de apoyo mutuo, por la autorreflexión y por 


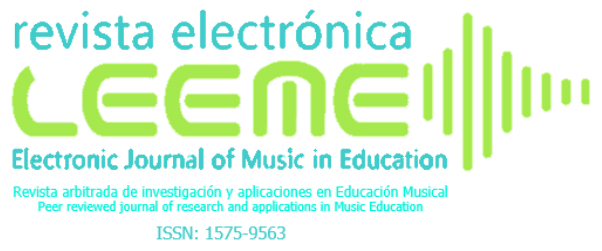

actitudes de participación voluntaria, de compromiso y de responsabilidad profesional. Todas estas particularidades del grupo docente conducen al desarrollo de un aprendizaje profesional compartido y a una cultura profesional comprometida. En resumen, al desarrollo profesional y a la mejora de la práctica docente y de la educación del alumnado (Montero, 2011).

En relación a las limitaciones del estudio, en su desarrollo se han planteado dificultades que a continuación se exponen:

- En el grupo de trabajo analizado no participan la totalidad del profesorado y alumnado de música de Primaria y Secundaria de centros públicos, concertados y privados de la ciudad de Manises.

- Este trabajo se ha planteado exclusivamente desde la perspectiva del profesorado.

- Por otro lado, la realización del presente estudio ha generado nuevas posibilidades a una futura investigación en la que se puede ampliar y dar continuidad al tema de investigación. A continuación, se muestran las propuestas de futuro que se tendrán en cuenta para seguir estudiando:

- Las percepciones de los diferentes integrantes de la comunidad educativa con respecto al CD: los estudiantes de $6^{\circ}$ de Primaria, como participantes protagonistas; la dirección de los centros docentes de Primaria y Secundaria, sobre las consecuencias de esta actividad musical en la vida escolar local; los profesores tutores y profesoras tutoras de los estudiantes de $6^{\circ}$ de Primaria, con respecto al proceso de transición musical desarrollado; y, por último, las familias del alumnado de $6^{\circ}$ de Primaria, en referencia a este recurso pedagógico musical como herramienta en la transición a Secundaria.

- El proceso de investigación-acción colaborativa del $\mathrm{CD}$, como modelo de innovación metodológica.

- La elaboración de un modelo de transición musical a través del CD que facilite la incorporación del alumnado de Primaria al instituto a través de un plan musical de acogida, implicando a toda la comunidad educativa.

Para terminar, se exponen las consideraciones finales de esta investigación, destacando especialmente tres aportaciones:

a). El presente estudio supone la presentación de una propuesta de acción docente en la que el profesorado de música de Primaria y Secundaria comparte con su alumnado una experiencia musical interactiva y significativa.

b). La participación activa en el proceso de organización del CD genera estrategias de aprendizaje colaborativo basadas en el consenso colectivo y en el análisis reflexivo, capaces de mejorar profesionalmente (Armengol, 2001). 


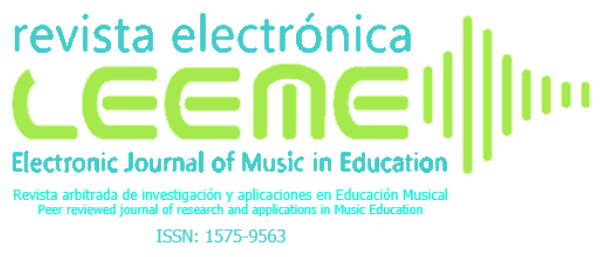

c). La coordinación docente mostrada en este estudio está caracterizada por afinidades personales y profesionales, por el compromiso por la educación, por la necesidad de evolucionar individual y colectivamente y por la satisfacción de conectar emocionalmente con el alumnado a través de la música.

En definitiva, todo lo mencionado anteriormente determina la coordinación y la colaboración docente de este grupo de docentes de Música que creen en este proyecto educativo musical, convirtiendo los centros educativos en espacios ilusionantes de trabajo, donde docentes y discentes se interrelacionan disfrutando de la magia y del valor de la música.

\section{Referencias}

Antúnez, A. (1999). El trabajo en equipo de los profesores y profesoras: factor de calidad, necesidad y problema. El papel de los directivos escolares. Educar, 24, 89-110. Recuperado de https://ddd.uab.cat/pub/educar/0211819Xn24/0211819Xn24p89.pdf

Armengol, C. (2001). La cultura de la colaboración. Reto para una enseñanza de calidad (p. 258). Madrid: La Muralla.

Cañizares, A.B., Pomares. E. y González, I. (2015). Profesorado de enseñanzas básicas de música de Andalucía: ¿mejoramos la coordinación docente? Eufonía: Didáctica de la Música, 63, 47-58.

Carrillo, C. y Vilar, M. (2014). El perfil profesional del profesorado de música: una propuesta de las competencias deseables en Ed. Primaria y Ed. Secundaria. Revista Electrónica de LEEME, 33, 1-26. Recuperado de https://ojs.uv.es/index.php/LEEME/article/view/9856

Castaño, T. (2012). Entre todos, para todos: el valor de hacer música en Secundaria. Eufonía: Didáctica de la Música, 56, 43-51.

Díaz, M. (2004). La música en la educación primaria y en las escuelas de música: la necesaria coordinación. Revista electrónica complutense de Investigación en educación musical, 1,1-14. Recuperado de https://revistas.ucm.es/index.php/RECI/article/view/9600

Escudero, J.M. (2008). Comunidades docentes de aprendizaje, formación del profesorado y mejora de la educación. Ágora para la EF y el Deporte, 10, 7-31. Recuperado de file:///C:/Users/Usuario/Downloads/DialnetComunidadesDocentesDeAprendizajeFormacionDelProfes-3048583\%20(1).pdf

Fabuel, V.S. (2015). Una reflexión sobre las transiciones educativas. De Primaria a Secundaria ¿traspaso o acompañamiento? EDETANIA, 48, 159-183. Recuperado de 
file:///C:/Users/Usuario/Downloads/Dialnet-

UnaReflexionSobreLasTransicionesEducativas-5349094.pdf

Fidalgo, M. y Barrera, A. (2014). La transición de la Primaria a la Secundaria. Ideas a partir de un estudio de caso [Mensaje en un blog]. Recuperado de: http://www.educaweb.com/noticia/2014/02/18/transicion-primaria-secundaria-ideaspartir-estudio-caso-8023/

Gimeno, J. (1996). La transición a la educación secundaria. Discontinuidades en las culturas escolares. Madrid: Morata.

González, C. y González, N. (2015). Enseñar a transitar desde la Educación Primaria: el proyecto profesional y vital. Revista Electrónica Interuniversitaria de Formación del Profesorado, 18, 29-41. doi: 10.6018/reifop.18.2.219291

Hargreaves, A. (1996). Profesorado, cultura y postmodernidad (Cambian los tiempos, cambia el profesorado). Madrid: Morata.

Hernández-Sampieri, R., Fernández, C. y Baptista, P. (2014). Metodología de la investigación. Sexta Edición. México: Mc Graw Hill.

Hurtado, J. (2004). Conciertos didácticos: aprendiendo a escuchar. Eufonía: Didáctica de la Música, 32, 51-59.

Hurtado, J. (2010). Los conciertos didácticos o la pluridimensionalidad de la educación musical: análisis comparativo de tres estudios de caso. En M.L. Martínez (Presidencia). I Congreso Internacional de Investigación en Música (pp.41-43). ISEACV: Valencia.

Hurtado, J. (2014). El concierto como estrategia aglutinadora de la experiencia musical. En J.L. Aróstegui (ed.), La Música en Educación Primaria. Manual de formación del Profesorado (pp.321-340). Madrid: Dairea.

Krichesky, G.J. y Murillo, F.J. (2011). Las comunidades profesionales de aprendizaje. Una estrategia de mejora para una nueva concepción de escuela. Revista Iberoamericana sobre Calidad, Eficacia y Cambio en la Educación, 9, 66-83. Recuperado de https://www.redalyc.org/pdf/551/55118790005.pdf

López, A. (2004). Colaboración y desarrollo profesional del profesorado. Regulaciones presentes en la estructura del puesto de trabajo (Tesis Doctoral). Universitat de València, València, España. 
Marcelo, C. (2001). Aprender a enseñar para la Sociedad del Conocimiento. Revista Complutense de Educación, 12, 531-593. Recuperado de C:IUsers\Usuario\Downloads\17605-Texto del artÃ-culo-17681

Marín, M.A. y Domínguez, I. (2015). Aprender por objetivos: un modelo de conciertos didácticos en la Fundación Juan March. Eufonía: Didáctica de la Música, 64, 29-38.

Martínez, M. y Pinya, C. (2015). La transición primaria-secundaria en los Institutos - Escuela en Catalunya. Revista Electrónica Interuniversitaria de Formación del Profesorado, 18, 57-72. doi: 10.6018/reifop.18.2.21914

Montero, L. (2011). El trabajo colaborativo del profesorado como oportunidad formativa. CEE Participación Educativa, 16, 69-88. Recuperado de http://www.ub.edu/obipd/docs/article_montero.pdf

Morant, R. (2014). Las Escuelas de Música de las Sociedades Musicales Valencianas. Valencia: Centro de estudios de la FSMCV.

Neuman, V. (2004). La formación del profesorado y los conciertos didácticos. Profesorado, Revista de Currículum y Formación del Profesorado, 8, 1-12. Recuperado de https://recyt.fecyt.es/index.php/profesorado/article/view/42108/24068

Pastor, P. (2004). Las marías.www.marchitopensil.boe.es. Revista Electrónica de LEEME, 14, $1-10$.

Pedrera, S. (2010). Análisis de algunas problemáticas específicas en la enseñanza y el aprendizaje musical en la educación secundaria obligatoria y el bachillerato. En A. Giráldez (coord.), Música. Investigación, innovación y buenas prácticas (pp.11-32). Barcelona: Graó.

Reyes, M. (2005). La música en educación primaria. Una perspectiva desde el maestro especialista (Tesis Doctoral). Universidad de Granada, Granada, España.

Ros, V. (2015). Los conciertos en el aula de educación musical de primaria: creación e interpretación cooperativa (Tesis Doctoral). Universitat de València, València, España.

Rusinek, G. (2002). Concierto de alumnos en el instituto. Eufonía: Didáctica de la Música, 25, 116-121.

Rusinek, G. (2004). Aprendizaje musical significativo. Revista Electrónica Complutense de Investigación en Educación Musical, 1, 1-16. Recuperado de file:///C:/Users/Usuario/Downloads/9622-Texto\%20del\%20art\%C3\%ADculo-9703-110-20110601.PDF 
Rusinek, G. (2008). Disaffected learners and school musical culture: an opportunity for inclusion. Research Studies in Music Education, 30, 9-23. doi: 10.1177/1321103X08089887

Sánchez, V. (2016). Los conciertos didácticos desde la mirada del programador. En M. Rodríguez (Presidencia). III Congreso Nacional Educación Musical, Almería.

Stake, R.E. (1998). Investigación con estudio de casos. Madrid: Morata.

@MARIA CAPEL MARTíN Y REMIGI MORANT NAVASQUILLO. THE CONTENT OF THIS ARTICLE IS THE SOLE RESPONSIBILITY OF THE AUTHORS. THE REVISTA ELECTRÓNICA DE LEEME AND UNIVERSITAT DE VALĖNCIA ARE NOT LABE FOR ANY LEGA ACTONS THAT MAY ARISE INVOLVING THE ARTICLE'S CONTENT. REVISTA ELECTRÓNICA DE LEEME - ISTA ELECTRÓNICA EUROPEA DE MÚSICA EN LA EDUCACIÓN-HTTP://OJS.UV.ES/INDEX/PHP/LEEME/INDEX. ISSN: 1575-9563. EDITORES: UNIVERSIDAD DE VALENCIA Y JESÚS TEJADA GIMÉNEZ. VISIBILIDAD DE ESTA REVISTA: SCOPUS, EMERGING SOURCES CITATION INDEX (CLARIVATE), EBSCO, CINDOC (CSIC), CITEFACTOR, COPAC, DIALNET, DICE (CSIC), DOAJ, E-REVISTAS (CSIC), EBSCO PREMIER, ERIH+, GALE CENGAGE LEARNING, IN-RECS, IRESIE, LATINDEX, MIAR, OCLC WORLDCAT, RESH, REDIB, RILM CORE JOURNALS, SUDOC, ULRICHS, ESTA REVISTA ESTÁ PUBLICADA CON EL APOYO INSTITUCIONAL DE REDIRIS-CONSEJO SUPERIOR DE INVESTIGACIONES CIENTIFICAS Y ES DE ACCESO LIBRE. CREATIVE COMMONS LICENSE 4.0 BY 